\title{
Electrical and mechanical probing of nanostructures with transmission electron microscopy
}

\author{
P. M. F. J. Costa ${ }^{*}$ Y. Bando** and D. Golberg** \\ * CICECO, Department of Chemistry, University of Aveiro, 3810-193 Aveiro, Portugal \\ ** MANA, National Institute for Materials Science, 305-0044 Ibaraki, Japan \\ Email: pedromfjcosta@ua.pt
}

Recent developments in the design of holders for transmission electron microscopes (TEM) have enabled widespread property analysis of nanostructures with unprecedented spatial resolution. It is now possible to routinely and reliably test the mechanical and electrical characteristics of a single carbon nanotube (CNT), for instance. Furthermore, due to the multitasking capability of TEMs, the study can even integrate simultaneous imaging and spectroscopical analysis. The combination of real-time gathering of structural, chemical, electrical and mechanical information provides an outstanding view of the materials' reactions when subjected to external stimulus such as current or heat.

Taking advantage of the aforementioned developments, we have been endeavouring to characterise a broad range of 1D nanostructures inside TEMs. One interesting example was the study of hybrid carbon nanotubes (Fig. 1), namely multi-walled CNTs filled with cooper iodide, CuI (a.k.a. CuI@MWCNT). Structural analysis showed that the encapsulated material consisted of chains of halide nanograins with $f c c$-type structure. Their growth orientation was irregular although a slight predominance of the relation [220]CuI//\{0002\}CNT was found. Moreover, several structural defects were present in the grains such as step edges and dislocations. The electrical behaviour of this material was followed with a scanning tunnelling microscopy (STM) device for TEMs [1]. It was observed that not only consistent Ohmic contacts can be achieved but also that the systems' conductance is dependent on the contents of the encapsulated halide which can be released with attogram $\left(10^{-18} \mathrm{~g}\right)$ precision. These effects could not be explained by variables such as the imaging electron beam, the nanotube-electrode contact area or even the morphology of the template nanotube. This work may help the development of tailored engineering of $1 \mathrm{D}$ nanostructures and their assimilation in nanoscaled delivery devices for medical, soldering or printing purposes.

Besides metals and halides, the electrical and mechanical behaviour of encapsulated optically active materials have also been explored [2]. A carbon nanotube filled with a doped II-VI semiconductor, $\mathrm{Zn}(\mathrm{Ga}) \mathrm{S}$, was subjected to compressive loads (Fig. 2). From the resulting force-displacement curves, measured with a purpose-built atomic force microscopy (AFM) holder for TEMs, it was possible to directly extract the Young's modulus for this material. Furthermore, by passing a high current flow through the structure, controlled discharge (partial or total) of the core filling was achieved. Reapplying compressive loads resulted in a remarkable altered behaviour, as illustrated in Fig. 2c. The empty section of the CNT would ultimately fail under the external stimulus leading to plastic deformation via the generation of kinks in the carbon structure. These took place exclusively in the empty portions of the nanotube. Since the mechanical properties of this hybrid CNT were so dependent of the presence of its core filling, this could provide the means to new technological applications such as nanosprings with "on-demand" variable stiffness. 


\section{References}

[1] P. M. F. J. Costa, D. Golberg, M. Mitome, S. Hampel, A. Leonhardt, B. Buchner, Y. Bando, Nano Letters, 8 (2008) 3120.

[2] P. M. F. J. Costa, U. K. Gautam, M. Wang, Y. Bando, D. Golberg, Carbon, 47 (2009) 527.

Project funded by the Nanotubes project, National Institute for Material Science.

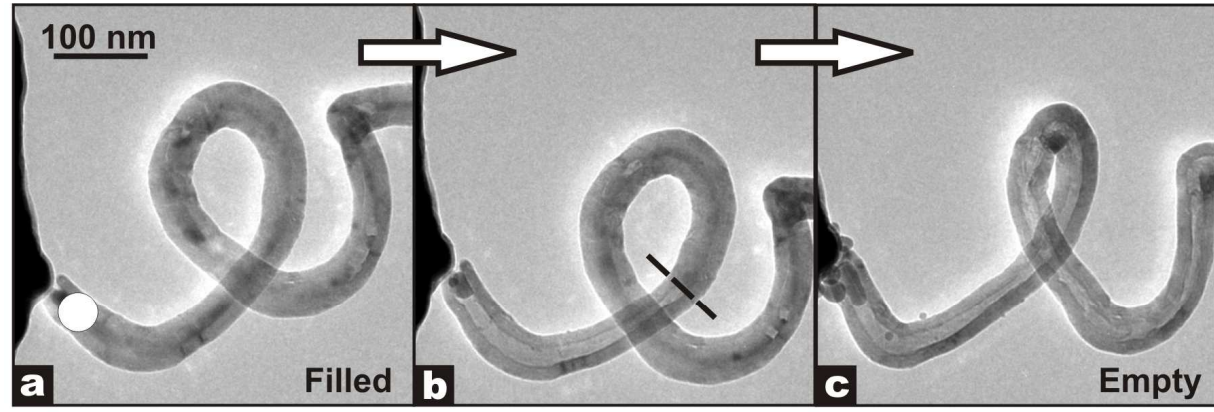

Fig. 1. (a to c) Sequential release of the core $\mathrm{CuI}$ filling from the inside of a coil-shaped carbon nanotube. (d) Energy dispersive X-ray spectrum of the circular white region marked in

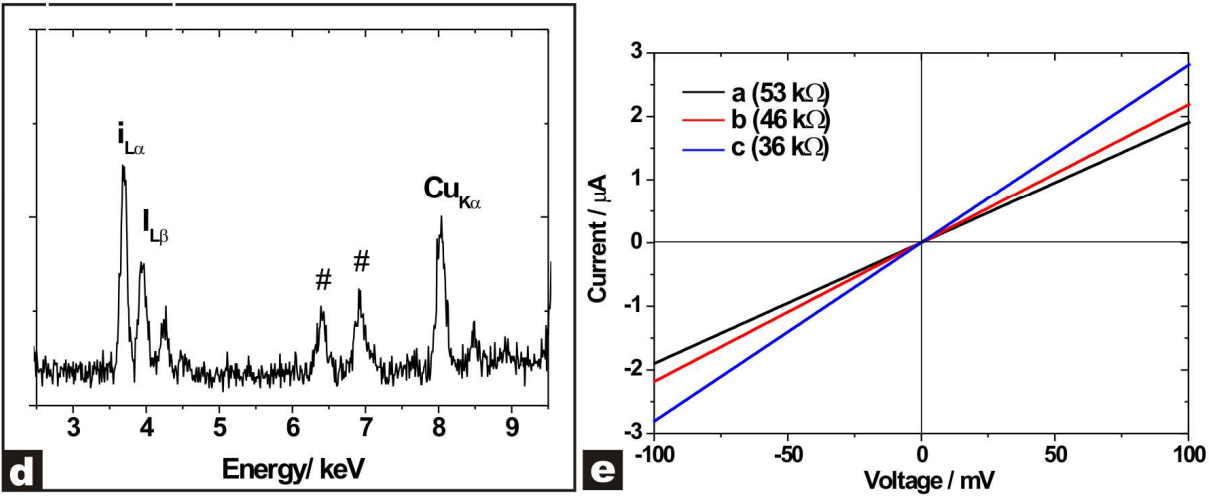
(a). (e) Current-voltage plots corresponding to stages a, b, c and respective total electrical resistance.

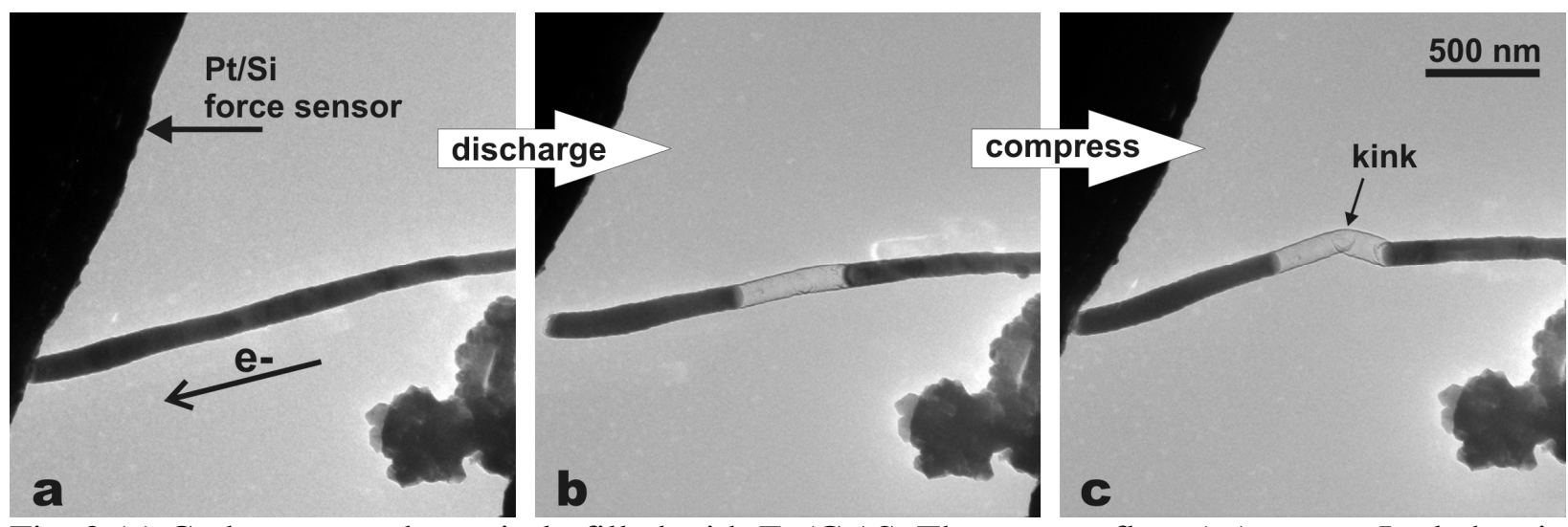

Fig. 2 (a) Carbon nanotube entirely filled with $\mathrm{Zn}(\mathrm{Ga}) \mathrm{S}$. The current flow (e-) causes Joule heating which leads to the discharge of the core material. (b) Nanotube seen in (a) after partial discharge of the core. (c) Plastic deformation of the structure in (b) when a compressive load was applied. 